\title{
Change of Resonant Electron Orbits from Trapped to Passing in Fast Wave Current Drive
}

\author{
Kun YAO and Yanping ZHAO \\ Institute of Plasma Physics, Chinese Academy of Sciences, Hefei, 230031, China
}

(Received 19 April 2010 / Accepted 19 May 2010)

\begin{abstract}
In fast wave current drive, fast waves accelerate resonant electrons in the direction parallel to the static magnetic field, causing parallel velocity to increase. The trajectory of a trapped resonant electron is calculated by computer code in which fast wave induced diffusion in velocity space is accounted for by a quasi-linear operator. Simulations show that the orbit of a trapped resonant electron transits from trapped to passing in some cases, reducing the effect of trapped electrons on current drive and improving current-drive efficiency. We determined the conditions under which this type of transition occurs.
\end{abstract}

(c) 2010 The Japan Society of Plasma Science and Nuclear Fusion Research

Keywords: fast wave current drive, resonant electron, electron orbit

DOI: $10.1585 /$ pfr.5.025

\section{Introduction}

A non-uniform magnetic field forms the magnetic wells in tokamak. These wells affect the motion of electrons with small parallel velocities relative to their perpendicular velocities by causing them to bounce along the field lines. These bounced electrons are called trapped electrons. In radio-frequency $(\mathrm{RF})$ current drive, trapped electrons may degrade current-drive efficiency because they absorb wave energy without producing net toroidal current in a time-averaged sense. Several researchers have studied the effect of trapped electrons on current-drive efficiency [1-6]. In kinetic theory, the bounce-averaging procedure enables us to consider the effect of trapped electrons on RF current drive [7-9].

In tokamak, a particle orbit can transit from one orbit class to the other. In ion cyclotron heating, as an ion passes through the cyclotron layer and undergoes random "kicks" [10], a continuous flow of particles transits from passing to trapped region and vice versa leading to ion radial diffusion [11]. In electron cyclotron resonance heating (ECRH), waves push electrons in a perpendicular direction, increasing the perpendicular velocity and causing the electron orbits to transit from passing to trapped. In fast wave current drive, the force exerted on electrons by a wave is in the parallel direction. If the force is large enough that a trapped electron can overcome the magnetic well, a resonant trapped electron can become a passing electron. In this paper, we show that a resonant trapped electron can become a passing electron when the diffusion coefficient is large enough.

author'se-mail: yaokun@ipp.ac.cn

\section{Simulation Model 2.1 Basic assumptions}

To prevent the important physical phenomena from being masked by possible computational and conceptual intricacies, we constructed the simplest prototype model that describes the resonant electron motion in fast wave current drive. The model neglects pitch-angle scattering and slowing-down collisions and considers the static electric field to be zero.

\subsection{Equilibrium magnetic field representa- tion}

The contravariant and covariant expressions of the equilibrium fields in an axisymmetric torus are

$$
\begin{aligned}
& B=\nabla \zeta \times \nabla \psi+q \nabla \psi \times \nabla \theta, \\
& B=g(\psi) \nabla \zeta+I(\psi) \nabla \theta+\delta(\psi, \theta) \nabla \psi,
\end{aligned}
$$

where $(\psi, \theta, \zeta)$ are the magnetic flux coordinates, $\psi$ is the poloidal magnetic flux, $\theta$ is the poloidal angle, and $\zeta$ is the toroidal angle.

In plasma equilibrium, the Grad-Shafranov equation can be written as

$$
R \frac{\partial}{\partial R}\left(\frac{1}{R} \frac{\partial \psi}{\partial R}\right)+\frac{\partial^{2} \psi}{\partial Z^{2}}=-\mu_{0} R J_{\phi}
$$

From (3), we can obtain the poloidal magnetic flux [12].

\subsection{Hamiltonian guiding center equations}

Electron trajectories are calculated by solving the following equations [13]:

$$
\begin{aligned}
& \frac{\mathrm{d} P_{\theta}}{\mathrm{d} t}=-\frac{\partial H}{\partial \theta}, \\
& \frac{\mathrm{d} \theta}{\mathrm{d} t}=\frac{\partial H}{\partial P_{\theta}},
\end{aligned}
$$




$$
\begin{aligned}
\frac{\mathrm{d} P_{\alpha}}{\mathrm{d} t} & =-\frac{\partial H}{\partial \alpha_{\mathrm{c}}}, \\
\frac{\mathrm{d} \alpha_{\mathrm{c}}}{\mathrm{d} t} & =\frac{\partial H}{\partial P_{\alpha}},
\end{aligned}
$$

where

$$
\begin{aligned}
& \frac{\partial Q(\psi, \theta)}{\partial \psi}=\frac{\partial \delta(\psi, \theta)}{\partial \theta}+\frac{\mathrm{d} q}{\mathrm{~d} \psi}, \\
& \alpha_{\mathrm{c}}=-\zeta+q \theta-\int_{P_{\alpha}}^{\psi}\left[\delta\left(\psi^{*}, \theta\right)+\frac{\mathrm{d} q}{\mathrm{~d} \psi} \theta\right] \mathrm{d} \psi^{*}, \\
& h(\psi)=q(\psi) g(\psi)+I(\psi), \\
& P_{\theta}=\rho_{/ /} h-\rho_{/ /} g Q+\int_{P_{\alpha}}^{\psi} Q\left(\psi^{*}, \theta\right) \mathrm{d} \psi^{*}, \\
& P_{\alpha}=\psi-\rho_{/ /} g, \\
& H=\frac{1}{2} \rho_{/ /}^{2} B^{2}+\mu B, \\
& \rho_{/ /}=\frac{m v_{/ /}}{B q}, \\
& \mu=\frac{1}{2} m \frac{v_{\perp}^{2}}{B} .
\end{aligned}
$$

\subsection{Diffusion operator}

We modeled the effect of fast wave on parallel velocity of a resonant electron with a Monte Carlo operator acting over time steps. The operator is given by [14]

$$
\Delta \lambda=-2\left(\lambda-\lambda^{3}\right) D \Delta t \pm \sqrt{\left(1-\lambda^{2}\right)^{2} D \Delta t},
$$

where

$$
\begin{aligned}
& \lambda=\frac{v_{/ /}-\left(v_{/ / 1}+v_{/ / 2}\right) / 2}{\left(v_{/ / 2}-v_{/ / 1}\right) / 2}, \\
& D=\left\{\begin{array}{l}
D_{0}, v_{/ / 1}<v<v_{/ / 2} \\
0, \text { otherwise }
\end{array}\right. \\
& D_{0}=\frac{\left\langle B_{/ /}^{2}\right\rangle}{B_{0}^{2}} k_{/ /}\left(2 v_{\mathrm{t}}^{2}-v_{\perp}^{2}\right)^{2} \pi \frac{4}{\left(v_{/ / 2}-v_{/ / 1}\right)^{3}},
\end{aligned}
$$

and where $\left\langle B_{\mid /}^{2}\right\rangle$ is the average square of the fast wave magnetic field, and $B_{0}$ is the equilibrium magnetic field on the axis. $v_{\mathrm{t}}$ is the electron thermal velocity and $k_{/ /}$is the mean wave number of the wave spectrum. The symbol \pm indicates that the sign is to be chosen randomly but with equal probability.

\section{Simulation Results and Discussion \\ 3.1 Simulation setup}

The wave amplitude and electron temperature profiles are of the form $\left(1-\rho^{2} / a^{2}\right)^{1 / 2}$ where $\rho$ is the half-width of a magnetic surface at the equator and $a$ is the minor radius. The plasma and fast wave parameters are listed in Table1. The equilibrium magnetic surfaces used in this paper are shown in Fig. 1. We simulated a typical test electron motion on a $129 \times 65(\psi, \theta)$ grids with the following initial settings: electron energy $10 \mathrm{keV}$, position $(1.9799 \mathrm{~m}$, $0.0000 \mathrm{~m}$ ), and pitch angle is $72^{\circ}$. The associated electron trajectory is shown in Fig. 2. The electron is deeply
Table 1 Simulations parameters.

\begin{tabular}{ll}
\hline Plasma parameters & Wave parameters \\
\hline$R_{0}=1.7 \mathrm{~m}$ & $v_{/ / 1}=10^{7} \mathrm{~m} / \mathrm{s}$ \\
$a=0.4 \mathrm{~m}$ & $v_{/ / 2}=10^{8} \mathrm{~m} / \mathrm{s}$ \\
$\kappa=1.88$ & $k_{/ /}=3 \mathrm{~m}^{-1}$ \\
$\delta=0.75$ & \\
$I_{\mathrm{p}}=1 \mathrm{MA}$ & \\
$B_{0}=3.5 \mathrm{~T}$ & \\
$T_{\mathrm{e} 0}=5 \mathrm{keV}$ & \\
\hline
\end{tabular}

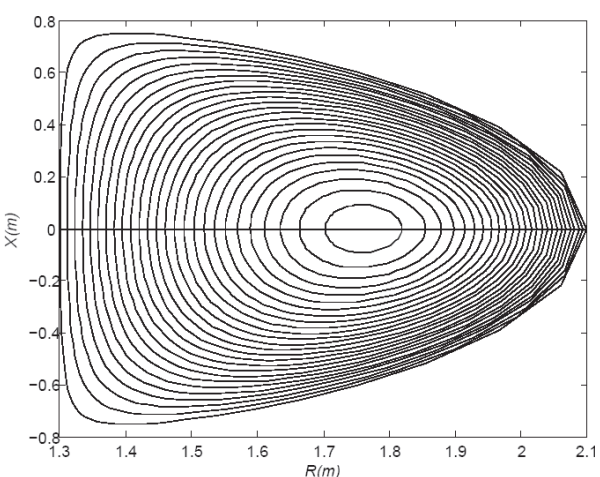

Fig. 1 Equilibrium magnetic surfaces.

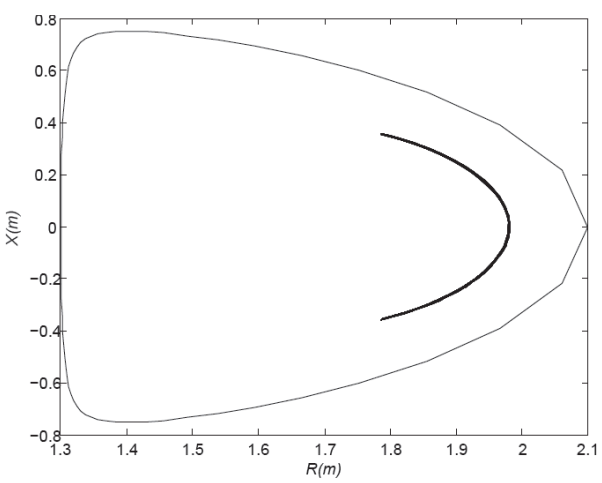

Fig. 2 Electron trajectory without fast wave.

trapped. The finite width of the trapped orbit is very small because the width is proportional to particle mass.

\subsection{Results and discussions}

We calculated electron trajectories for different wave amplitudes $\bar{B}_{/ /}=\sqrt{\left\langle B_{/ /}^{2}\right\rangle}$. Trajectories are shown for three cases in Fig. 3. For the first two cases (Figs. 3 (a) and (b)), the trapped resonant electron changes to a passing electron because the fast wave amplitude is large, the resonant electron orbit changes from trapped to passing faster for the first case than for the second case after 1000 and 5000 time steps, respectively, where a time step is $10^{-8} \mathrm{~s}$. For the third 


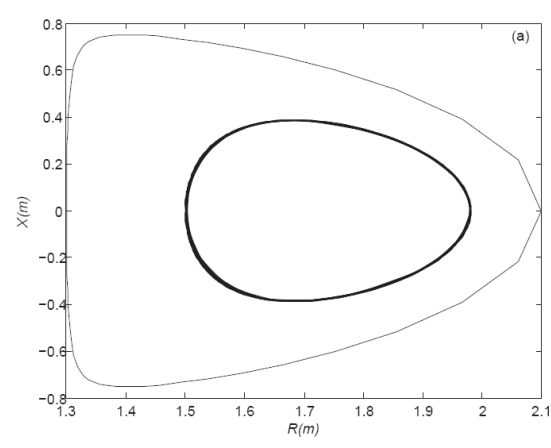

(a) $\bar{B}_{/ /} / B_{0}=0.01$

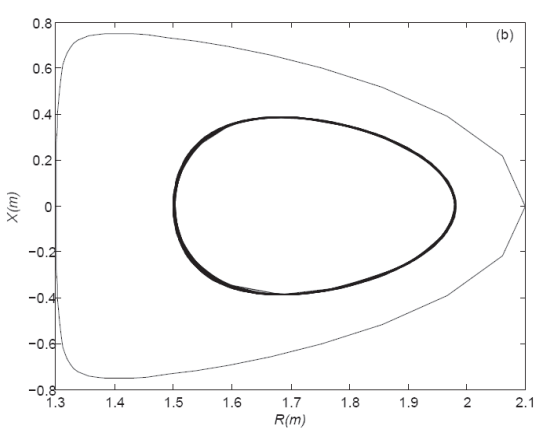

(b) $\bar{B}_{/ /} / B_{0}=0.001$

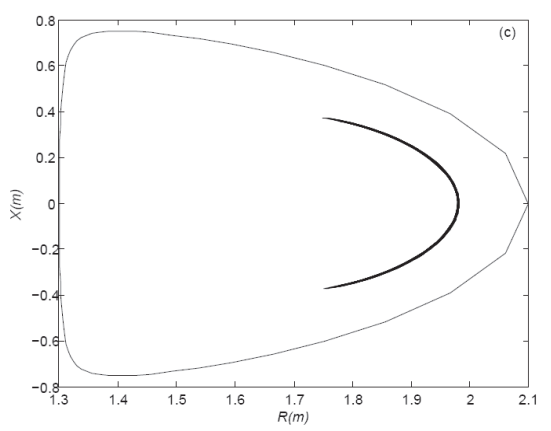

(c) $\bar{B}_{/ /} / B_{0}=0.0001$

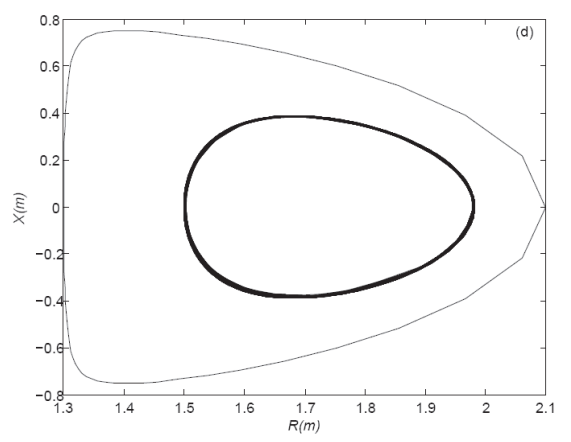

(d) $\bar{B}_{/ /} / B_{0}=0.0001$

Fig. 3 Electron trajectories with different fast wave amplitudes. case (Fig. 3 (c)), the fast wave amplitude is so small that the trapped electron cannot overcome the magnetic well after 10000 time steps.

The force equation for resonant electrons is [6]

$$
\begin{aligned}
& m_{\mathrm{e}} \frac{\mathrm{d} v_{/ /}}{\mathrm{d} t}=\left\langle F_{\mathrm{w}}\right\rangle+\left\langle F_{\mathrm{g}}\right\rangle, \\
& F_{\mathrm{w}}=\frac{e}{2 \Omega_{\mathrm{e}}}\left(2 v_{\mathrm{t}}^{2}-v_{\perp}^{2}\right) \frac{\partial B_{/ /}}{\partial z}, \\
& F_{\mathrm{g}}=-\mu \nabla_{/ /} B,
\end{aligned}
$$

where $F_{\mathrm{w}}$ is the force exerted on an electron by a fast wave, and $F_{\mathrm{g}}$ is the force arising from the gradient of the static magnetic field. \langle\rangle denotes time average. In a bounce pe$\operatorname{riod} T_{\mathrm{b}},\left\langle F_{\mathrm{g}}\right\rangle$ is about zero. After time $t=n T_{\mathrm{b}}$, the increase in parallel velocity is

$$
\Delta v_{/ /}=\frac{\left\langle F_{\mathrm{w}}\right\rangle}{m_{\mathrm{e}}} t .
$$

The trapped electron satisfies the condition

$$
\frac{B_{\min }}{B_{\max }}<\left(\frac{v_{0 \perp}}{v_{0}}\right)^{2}
$$

As parallel velocity increases, the trapped resonant electron becomes a passing electron and satisfies the condition

$$
\frac{B_{\min }}{B_{\max }} \geq \frac{v_{0 \perp}^{2}}{v_{0}^{2}+\left(\Delta v_{/}\right)^{2}} .
$$

From (25), we obtain

$$
t \geq \sqrt{\frac{v_{0 \perp}^{2}-v_{0}^{2} B_{\min } / B_{\max }}{B_{\min }\left\langle F_{\mathrm{w}}\right\rangle^{2} /\left(B_{\max } m_{\mathrm{e}}^{2}\right)}} .
$$

Obviously, when time is long enough, a trapped resonant electron becomes a passing electron regardless of the fast wave amplitude. In the third case, from (26) the detrapping time is about $0.3 \times 10^{-3} \mathrm{~s}$. If we increase the time for simulation, a trapped electron changes to a passing electron after 100,000 time steps, because the sum of 100,000 time steps is larger than the detrapping time as shown in Fig. 3 (d). However, if the perpendicular velocity is large and satisfies the condition

$$
\frac{B_{\min }}{B_{\max }}<\frac{v_{0 \perp}^{2}}{v_{0 \perp}^{2}+v_{/ / 2}^{2}},
$$

then the trapped resonant electron cannot change to a passing electron. If a resonant electron is accelerated by a fast wave, its parallel velocity can be out of the resonant velocity range, so that it is no longer accelerated by the fast wave. In fast wave current drive, Landau damping and transit time magnetic pumping induce two opposite parallel forces. When $v_{\perp}^{2}=2 v_{\mathrm{t}}^{2}$, the two forces exactly cancel and the diffusion coefficient is zero. Therefore a trapped resonant electron with $v_{\perp}^{2}=2 v_{\mathrm{t}}^{2}$ cannot become a passing electron. 


\section{Summary and Conclusions}

In the outer part of a magnetic surface, the inverse aspect ratio is not small and a large fraction of electrons are trapped. These trapped electrons degrade the fast wave current drive efficiency. Simulations show that, in fast wave current drive, a fraction of the trapped resonant electrons becomes passing electrons after a period of time. In actual situations, the current drive time is finite. If the fast wave amplitude is very small, a trapped resonant electron will not become a passing electron within the current drive time. To reduce the effect of trapped electrons on current drive, we need to prolong the current drive time, enhance the power of the fast wave, and raise the upper limit of the resonant velocity region.

\section{Acknowledgements}

The author thanks Xiao for the assistance provide with computer code and for the helpful discussions.
[1] G. Giruzzi, Nucl. Fusion 27, 1934 (1987).

[2] R.H. Cohen, Phys. Fluids 30, 2442 (1987).

[3] D.A. Ehst, Nucl. Fusion 31, 1933 (1991).

[4] J.G. Cordey, T. Eddlington and D.F.H. Start, Plasma Phys. 24, 73 (1982).

[5] S.C. Chiu, V.S. Chan, R.W. Harvey et al., Nucl. Fusion 29, 2175 (1989).

[6] N.J. Fisch and C.F.F. Karney, Phys. Fluids 24, 27 (1981).

[7] G.D. Kerbel and M.G. McCoy, Phys. Fluids 28, 3629 (1985).

[8] D.A. Ehst and K. Evans, Nucl. Fusion 26, 461 (1986).

[9] R. Biato, M. Brambilla, I. Pavlenko et al., Nucl. Fusion 42, 1085 (2002).

[10] T.H. Stix, Nucl. Fusion 15, 737 (1975).

[11] W.G.F. Core, Nucl. Fusion 29, 1101 (1989).

[12] S.B. Zheng, A.J. Wootton and R.E. Solano, Phys. Plasmas 3, 1176 (1996).

[13] S.J. Wang, Phys. Plasmas 13, 052506 (2006).

[14] S.K. Sipila and J.A. Heikkinen, IEEE Trans. Plasma Sci. 22, 260 (1994). 